\title{
Pattern Doping into Non-Substituted Poly( $p$-phenylene vinylene) by a Simple Vacuum Process for a Multicolored Luminescence Medium
}

\author{
Hiroyuki MochizUKI, ${ }^{\dagger}$ Toshiko MizoKURo, Nobutaka TANIGAKI, \\ Xeliang Mo, and Takashi HIRAGA \\ Photonics Research Institutes, National Institute of Advanced Industrial Science and Technology, \\ 1-8-31 Midorigaoka, Ikeda 563-8577, Japan
}

(Received August 18, 2005; Accepted September 2, 2005; Published January 15, 2006)

\begin{abstract}
Functional dyes could be dispersed into non-substituted poly( $p$-phenylene vinylene) (PPV), which shows a poor processability due to its insoluble and infusible, by the vacuum process under the thermal equilibrium state without the structural damage of PPV. Using this methods, it was possible to change the color of PPV from yellow to green with the blue dye, 1,4-( $N, N^{\prime}$-diethylamino)anthraquinone (SV59). The amount of SV59 doped into PPV increased with temperature and treatment time. The fluorescence color of PPV could be changed from green to red by 2 min doping of 4-(dicyanomethylene)-2-methyl-6-(4-dimethylaminostyryl)-4H-pyran (DCM). Furthermore, a pattern doping with DCM could be carried out resulting in production of multicolored luminescent medium.
\end{abstract}

[DOI 10.1295/polymj.38.73]

KEY WORDS Vapor / Dye / Poly(p-phenylene vinylene) / Doping /

Conjugated or 'conducting' polymers are currently attracting significant interest as materials for a wide variety of applications, including those making use of their electronic and optical properties. ${ }^{1-7}$ Among the $\pi$-conjugated polymers known, poly ( $p$-phenylene vinylene) (PPV) has been used in electronic devices such as an electroluminescence device ${ }^{8,9}$ and a solar cell $^{10}$ and exhibits remarkable chemical and thermal stability. However, PPV is insoluble and infusible, so shows poor processability. For the non-substituted $\pi$-conjugated polymers including PPV, doping is a promising, important way to enhance and control their properties.

There have been previous reports of doping in PPV. In the 1980 's, it was reported that dopings with $\mathrm{I}_{2}$, $\mathrm{AsF}_{5}$, and $\mathrm{SO}_{3}$ under reduced pressures of 3-350 $\mathrm{mmHg}$ could increase the conductivities of PPV. ${ }^{11}$ This is a conventional method for doping in $\pi$-conjugated polymers and is acceptable for dopants showing relatively high vapor pressures and small molecular sizes. However, it is difficult to disperse dopants showing relatively low vapor pressures and large molecular sizes such as organic dyes. PPV doping with tris(8-hydroxyquinoline)aluminum $\left(\mathrm{Alq}_{3}\right)$ was carried out by the same method. ${ }^{12}$ However, $\mathrm{Alq}_{3}$ was "deposited" onto the PPV surface but not within PPV. ${ }^{12,13}$

Solution processes of the PPV doping have been also reported. At present, PPV can be produced conveniently by a two-step indirect synthesis, i.e., prepared from a sulfonium salt precursor polymer ${ }^{14}$ and the precursor route has been used widely for the syn- thesis of high molecular weight PPV. As mentioned above, PPV is insoluble and infusible, but the sulfonium salt precursor polymer is water-soluble. Park and co-workers built a photoconductive device using a composite system of PPV and viologen dibromide, ${ }^{15}$ which was prepared from a mixture of the sulfonium salt precursor polymer and viologen. However, this method can be applied for the photoconductive devices, but not favorable for devices requiring good film-forming ability and nonexistence of aggregation, namely, uniform dispersal of an additive. Therefore, the development of a standard and easy method of doping into PPV is important for device fabrication using PPV, and PPV can not show adequately its potential performance.

We have developed a doping method termed the "vapor-transportation method" with a vacuum technique. ${ }^{16-21}$ This method vaporizes molecules of a dye, which then permeate into a polymer under vacuum $\left(\sim 10^{-6} \mathrm{~Pa}\right.$, much higher vacuum than the conventional method) after sublimation of the dye by heating in a vacuum. In this method, the entire vacuumed vessel is heated to be under thermal equilibrium, while in the conventional method only the dopant is heated or all treatments occur at room temperature.

In the present study, we report that the vapor-transportation method can be applied for dye-doping in PPV and can readily achieve molecular dispersal of functional dyes in PPV (i.e., the dye are not deposited onto the PPV surface) without deformation of the initial shapes. Furthermore, dispersal behavior of doping

${ }^{\dagger}$ To whom correspondence should be addressed (E-mail address: h-mochizuki@aist.go.jp). 


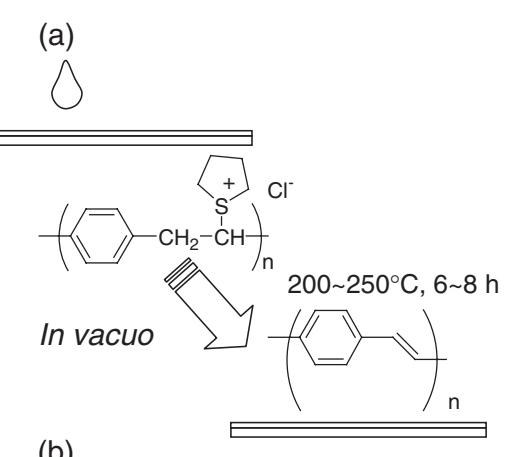

(b)

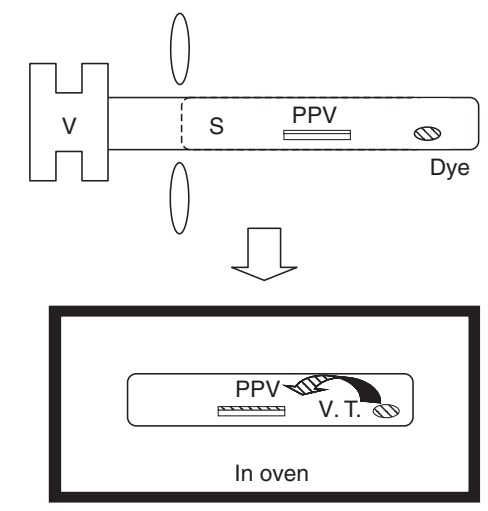

Figure 1. (a) Illustrations of the formation of PPV and chemical structures of PPV and the precursor. (b) Sample preparation by the vapor transportation method. V, vacuum pump; S, sealing; V. T., vacuum transportation.

into PPV was evaluated and the pattern doping could be performed resulting in the fabrication of multicolored luminescent medium.

\section{EXPERIMENTAL SECTION}

Figure 1 illustrates a schematic representation of sample preparation and the chemical structure of a precursor and PPV. PPV films on quartz glass plates were prepared by thermal elimination of the cast or spin-coated films from a $1 \mathrm{wt} \%$ aqueous solution of the precursor polymer (poly $(p$-xylene tetrahydrothiophenium chloride); see Figure 1a) of PPV. The $1 \mathrm{wt} \%$ aqueous solution was obtained from dilution of a $2.5 \mathrm{wt} \%$ aqueous solution of the precursor polymer purchased from Aldrich Co., Ltd. The thermal elimination for formation of the PPV film was performed at $200-250^{\circ} \mathrm{C}$ for $6-8 \mathrm{~h}$ in vacuo. The resulting PPV film on the quartz glass plate was loaded in a glass tube with a sufficient amount of dye (10-100 $\mathrm{mg}$ ). The pumped pressure in the tube was reduced by using a turbo molecular pump as shown in Figure 1b. After reaching an optimum pressure of around $10^{-6} \mathrm{~Pa}$, the glass tube was sealed by melting to form an ampoule. The ampoule was set at a constant temperature in an oven as a thermal treatment, then cooled down slowly over $2 \mathrm{~h}$ to room temperature.

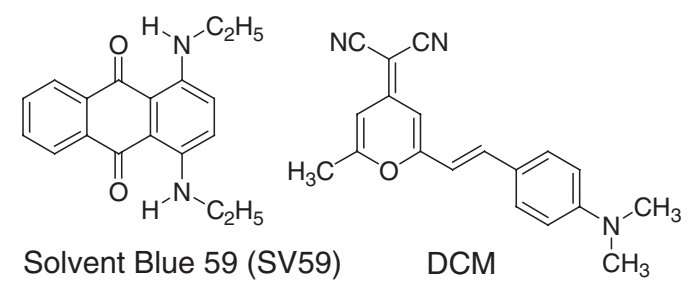

Figure 2. Chemical structures of the dyes used in this study. SV59 and DCM are a usual employed blue dye and a well-known red laser dye, respectively.

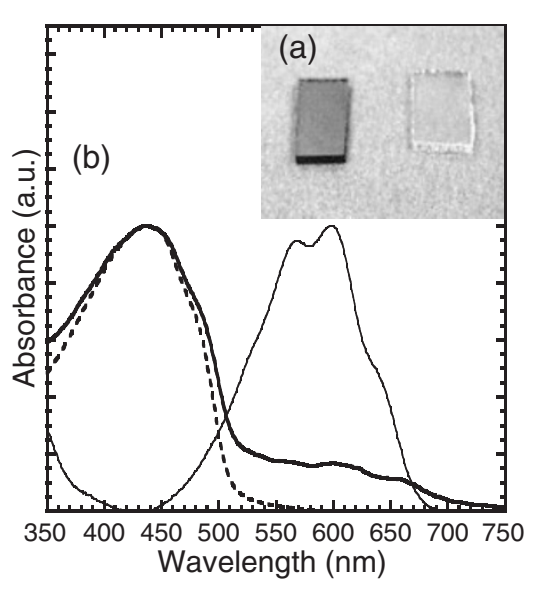

Figure 3. (a) Photograph of PPV (right) and SV59-doped PPV (left). Photographs were taken after wiping the surface of SV59doped PPV film with ethanol or acetone. (b) Absorption spectra of PPV (thicker dash line) and SV59-doped PPV (thicker solid line). Thinner solid line indicates an absorption spectrum of SV59 in 1,2-dichloroethane.

In this study, doped materials were 1,4-( $N, N^{\prime}$-diethylamino)anthraquinone (SV59) and 4-(dicyanomethylene)-2-methyl-6-(4-dimethylaminostyryl)-4H-pyran (DCM). SV59 and DCM were also obtained from Aldrich Co., Ltd. and used after sublimation purification. SV59 and DCM are usually employed as a famous blue dye and a well-known laser dye, respectively. The chemical structures of these dyes are shown in Figure 2.

Measurements of absorption and fluorescence spectroscopies were performed with an absorption spectrometer of Shimadzu UV-3150 and a fluorescence spectrometer of Hitachi F-4500, respectively.

\section{RESULTS AND DISCUSSION}

\section{Doping of SV59 into PPV}

Figure 3 a shows the PPV film on the substrate and the PPV film doped with SV59 (SV59/PPV), both films were $\sim 200 \mathrm{~nm}$ thick. The latter film was prepared by dispersal of SV59 at $150{ }^{\circ} \mathrm{C}$ for $5 \mathrm{~h}$. The PPV film was clearly yellow in color, while the SV59/PPV film was green. By dispersal of blue dye SV59, color 
of the film changed from yellow to green, indicating a color mixture of yellow and blue. In this method, color shading, deformation, peeling and roughness did not occur in the doped film. After wiping the doped film with an ethanol and an acetone, no changes were observed in the color of the wiping cloth or the surface of the doped PPV. Thus, SV59 did not sit on the surface but dispersed within the PPV. Absorption spectra of the SV59/PPV film and the PPV film are revealed in Figure 3b. PPV shows a maximum absorption at around $450 \mathrm{~nm}$ and an absorption edge at less than $570 \mathrm{~nm}$. The SV59/PPV film showed an absorption peak at the longer wavelength of $570 \mathrm{~nm}$, which was not observed in undoped PPV. Furthermore, SV59/ PPV showed the same absorption maximum as nondoped PPV. If doping of SV59 affects the structure of PPV (for example, promotion of crystallization, complex formation, chemical reaction, pyrolysis, and so on), the absorption maximum should be shifted. In the present study, as there was no shift in absorption maximum of PPV, it was considered that there was no significant effect of SV59 doping on the structure of PPV. As PPV is a semi-crystalline polymer, ${ }^{22,23}$ it contains some amorphous regions, so SV59 molecules seem to perform easier percolation into the amorphous regions in PPV. In the present method, the vaporized molecules with thermally assisted molecular motion came into contact with the PPV surface heated. So, the dye molecules were assumed to disperse within the PPV showing insolubility. From our result that the amount of dye doped into polystyrene were markedly higher than those into poly(methyl methacrylate), ${ }^{19}$ the dye molecules could readily coordinate the benzene ring of the polymer such as so-called " $\pi-\pi$ interaction" ${ }^{24-26}$ as in SV59 and PPV.

To quantify SV59 dispersed in PPV, changes in the weight after treatment $\left(150^{\circ} \mathrm{C}, 5 \mathrm{~h}\right)$ were analyzed, that is, the amount of SV59 was evaluated from increases in the film weight of many samples and these were dealt statistically. The resulting amount of SV59 dispersed in PPV was $\sim 2.7 \mathrm{wt} \%$.

\section{Dispersion Behavior of SV59 into PPV}

Evaluation of dispersion behavior of SV59 in PPV was performed using a $150 \mathrm{~nm}$ thickness PPV film by absorption spectroscopy. As indicated in Figure 3b, the absorption of SV59 does not exist at around 430 $\mathrm{nm}$ where the absorption maximum of PPV shows. PPV does not possess its absorption larger than 570 $\mathrm{nm}$ where there is an absorption maximum of SV59, namely, absorption maxima of PPV and SV59 are unrelated, independent to each other. Thus, the dispersal behavior of SV59 can be investigated by absorption ratio $\left(\mathrm{A} / \mathrm{A}_{\mathrm{P}}\right)$, where $\mathrm{A}$ and $\mathrm{A}_{\mathrm{P}}$ are the absorption max-

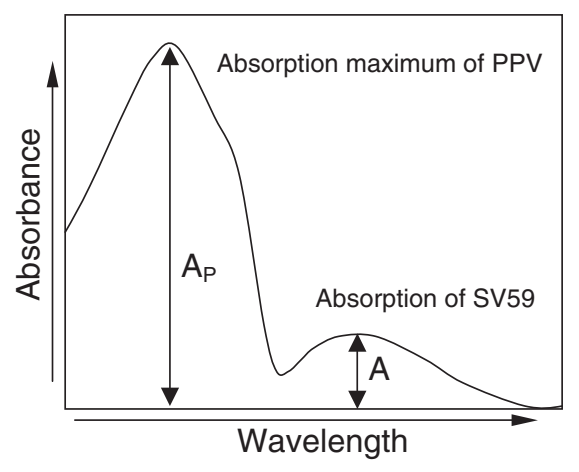

Figure 4. (a) Evaluation of dispersion behavior of SV59 in PPV. Absorption maxima of PPV and SV59 were independent. Dispersion behavior was estimated as the absorption ratio of $A / A_{P}$.
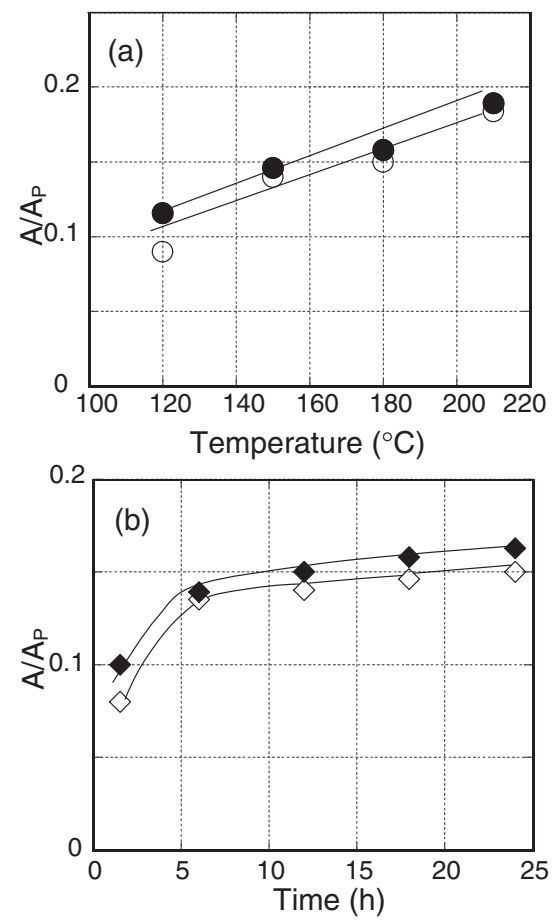

Figure 5. (a) Data plots of $A / A_{P}$ against temperature; closed circle and open circle denote treatment time of 18 and $12 \mathrm{~h}$, respectively. (b) Data plots of A/AP against treatment time: closed rhombus, treatment at $180^{\circ} \mathrm{C}$; open rhombus, treatment at $150{ }^{\circ} \mathrm{C}$.

imum of SV59 at $600 \mathrm{~nm}$ and PPV at $430 \mathrm{~nm}$, respectively (see Figure 4), and a rise in $\mathrm{A} / \mathrm{A}_{\mathrm{P}}$ means an increase in the doping amount of SV59 in PPV. Figure 5a displays the $\mathrm{A} / \mathrm{A}_{\mathrm{P}}$ value as a function of temperature. In this figure, it was found that the $\mathrm{A} / \mathrm{A}_{\mathrm{P}}$ value was observed even at $120^{\circ} \mathrm{C}$. In cases of treatment time of both 12 and $18 \mathrm{~h}$, the $\mathrm{A} / \mathrm{A}_{\mathrm{P}}$ value increased simply as temperature rose. As a sublimated pressure rises with temperature, more vaporized molecules of SV59 exist in the vacuum ampoule with temperature, and more molecules come into contact with the PPV surface. Furthermore, treatment even at $210^{\circ} \mathrm{C}$ did not damage thermally and did not affect morphology of the doped film. Next, plots of $\mathrm{A} / \mathrm{A}_{\mathrm{P}}$ against treat- 
ment time are shown in Figure 5b. It appears that the $\mathrm{A} / \mathrm{A}_{\mathrm{P}}$ value increases sharply until treatment time of $5 \mathrm{~h}$ and grows slightly from $5 \mathrm{~h}$ in case of both treatment temperatures of 150 and $180^{\circ} \mathrm{C}$. It was thought that the head region of dye dispersion took $6 \mathrm{~h}$ to reach the other substrate (glass substrate) indicating larger slope in Figure 5b. After reach of the head region, dispersal amount increased slowly and saturated. As described above, since thickness of the PPV film was $\sim 150 \mathrm{~nm}$, it took shorter time to reach a saturated state than our expectation (expected that saturation time was over $24 \mathrm{~h}$ ). We tried to observe the dispersion behavior with electron probe micro analyzer. However, the PPV film was so thin that we could not obtain a clear result.

\section{Doping of DCM into PPV}

Figure 6a indicates a photograph of non-doped PPV and DCM-doped PPV (DCM/PPV) irradiated with a UV lamp at $365 \mathrm{~nm}$. Both films were $\sim 150 \mathrm{~nm}$ thick. The DCM/PPV film was prepared by exposure to $\mathrm{DCM}$ at $180^{\circ} \mathrm{C}$ for $15 \mathrm{~min}$. It is clear that the nondoped PPV film emits green fluorescence, while the DCM/PPV film showed bright and uniform fluorescence in the orange-red region. Here, evaluation of fluorescence spectroscopy for these films was performed and the results are shown in Figure 6b. On

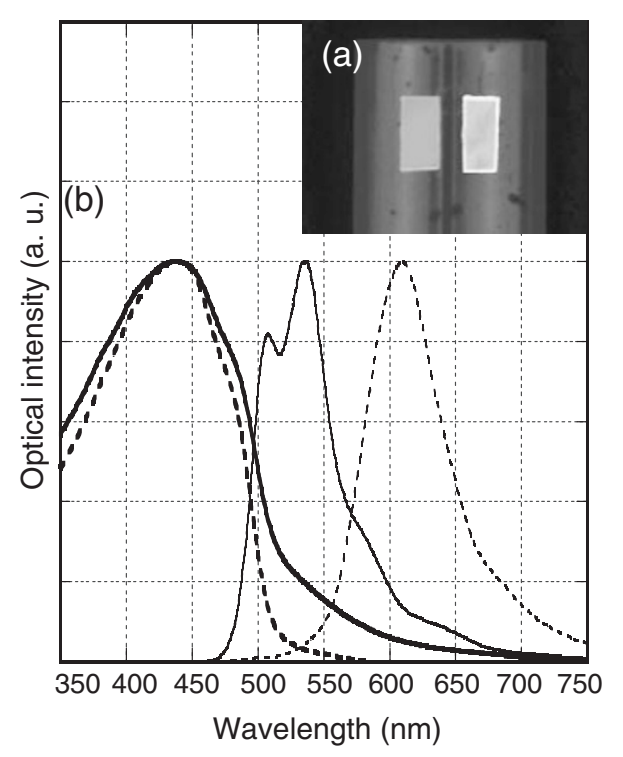

Figure 6. (a) Photograph of PPV and DCM-doped PPV under irradiation at $365 \mathrm{~nm}$ on the polymer side. PPV emitted blue-green fluorescence, while doped PPV showed red-orange fluorescence. (b) Absorption and fluorescence spectra of PPV and DCM/PPV. Thicker dash and solid lines indicate the absorption spectra of PPV and DCM/PPV, respectively, and thinner solid and dash lines denote the fluorescence spectra of PPV and DCM/PPV, respectively. Similar to the absorption of SV59/PPV, DCM-doped PPV showed the same absorption maximum as non-doped PPV. Excitation wavelength was $370 \mathrm{~nm}$ in both samples. irradiation at $370 \mathrm{~nm}$, non-doped PPV film showed a fluorescence spectrum with two maxima at around 510 and $530 \mathrm{~nm}$. The DCM/PPV films did not show any fluorescence maxima at around 510 and $530 \mathrm{~nm}$, but exhibited a maximum at around $610 \mathrm{~nm}$, which was nearly the same as the fluorescence spectrum of DCM, suggesting that DCM quenched the emission of PPV in DCM/PPV. As there is also absorption of $\mathrm{DCM}$ at $370 \mathrm{~nm}$, if the energy transfer did not occur, the DCM/PPV film should show the PPV fluorescence maxima (at around 510 and $530 \mathrm{~nm}$ ) and the DCM maximum (at around $610 \mathrm{~nm}$ ). However, as the assumed fluorescence was not observed, these results indicate the occurrence of energy transfer between PPV and DCM and the lack of complex formation between PPV and DCM. Detailed experiments indicated that the fluorescence of PPV was quenched completely, i.e., was changed from green to red, by only 2 min exposure to DCM. Furthermore, the properties of PPV doped by our method, such as color and fluorescence, remained unchanged for over a year (The doped PPV after a year showed the same spectrum as that doped just), since dyes can not depart from the doped PPV film at room temperature in the atmosphere (in a desiccator in our laboratory).

\section{Pattern Doping of DCM into Non-substituted PPV}

Patterning is of importance in many regions of modern science and technologies, with applications ranging from the production of the integrated circuits, information storage devices, and display units to the fabrication of micro-electromechanical systems. Particularly for opto-electronic devices based on the $\pi$ conjugated polymers, these are dependent on the construction fabricated by patterning process. ${ }^{27}$ Thus, we tried to dope DCM in patterned areas of PPV. A schematic representation for a patterned PPV film is shown in Figure 7. Patterned aluminum (Al) depositions were performed through a mask on the PPV films, which were coated onto a quarts substrate whose size was $7 \times 4 \times 1 \mathrm{~mm}^{3}$. In the method motioned here, as the vaporized dye filled in the ampoule and was allowed to diffuse through a small opening, the patterning needs the gapless mask like an $\mathrm{Al}$ deposition. After the $\mathrm{Al}$ deposition, DCM was doped by the vapor transportation method at $180^{\circ} \mathrm{C}$ for $30 \mathrm{~min}$. DCM could not be dispersed in the Al-deposited area but be doped in the PPV area. After DCM doping, Al was over-deposited onto whole surface in order to equalize the emission condition. Figure 8 indicates photographs of the samples irradiated at $366 \mathrm{~nm}$ at the side of the quarts substrate (see upside of Figure 8). The doped area of DCM emitted a bright red-orange luminescence and the non-doped area showed a light green emission. The doped PPV film in the present 


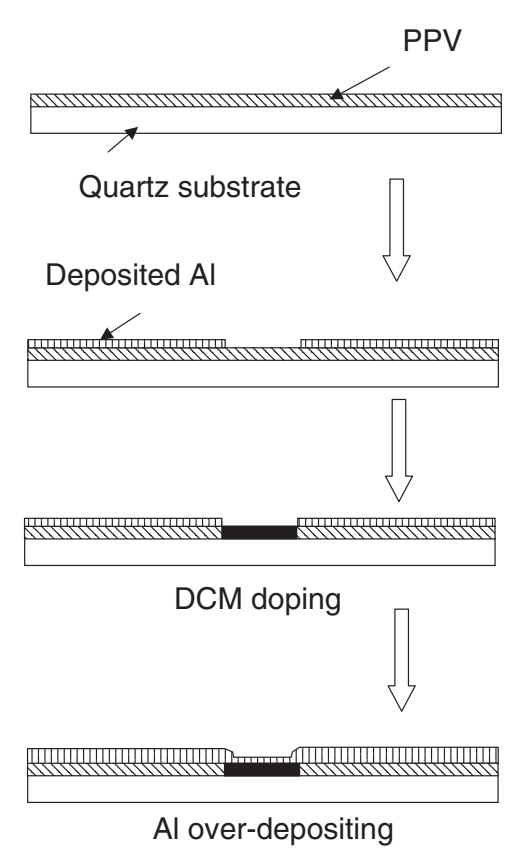

Figure 7. A schematic representation for pattern doping of DCM.
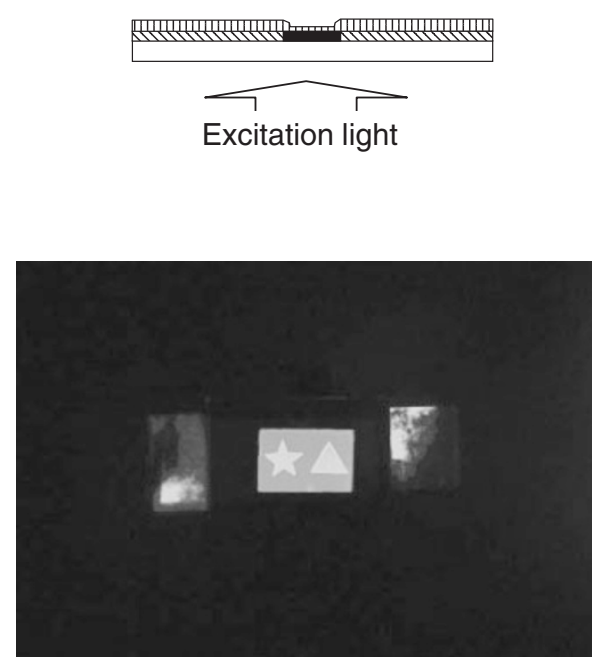

Figure 8. Photographs of a medium, which were carried out pattern doping of DCM, irradiated at $366 \mathrm{~nm}$ at a front side.

study was confirmed to emit a multicolored photoluminescence. As irradiated at side of the quarts substrate, irradiation light was absorbed almost in an interface-neighborhood between the glass substrate and PPV, so that the luminescence emitted in the interface-neighborhood of PPV. Since the result that the fluorescence color changed from green to red due to the fluorescence of DCM, DCM seemed to penetrate within PPV and to reach the interface.

Among fabrication processes of multicolored luminescent devices, a spin-coat process is usual because of ease in the laboratory. However, the spin coat process is difficult to perform a pattern coating onto the substrate without a surface treatment. It is unable to make a multi-layered structure by spin-coat because a polymer solution affected the pre-formed polymer layer. Before everything, the spin coat process cannot use non-substituted PPV due to its insolubility. On the other hand, the ink jet method is another process as a fabrication system for multicolored device, ${ }^{28,29}$ but it requires luminescent dyes, a patterned substrate, the precious system, and soluble PPVs.

\section{CONCLUSIONS}

Substituted PPVs have been developed because of addition of solubility into PPV and are easy mixing of functional, nonionic compounds due to its solubility for organic solvent. ${ }^{30-32}$ However, substituted PPVs are based on difficult preparations owing to many synthesis steps. In the view of the hard preparation of soluble PPV by the difficult syntheses, it is necessary to explore a novel doping method for enhancement of PPV characteristics. We showed here the "vapor transition method" as a novel doping method, and functional insolubility polymer, such as PPV, could be dispersed active compounds by this method. Generally, in doping, a small amount of dopant can induce a large change in characteristic and can markedly enhance the properties of polymers. The method described here seemed also to disperse a small quantity of dye, but enabled us to easily change the color and fluorescence of PPV films. Furthermore, a multicolored luminescent medium was fabricated through the mask process. Thus, this method is a good alternative for the doping process because of its simplicity and versatility, and may be acceptable for other insoluble $\pi$-conjugated polymers. The results of the present study suggested that the vapor transportation method might be useful for fabrication of electroactive devices using PPV.

Acknowledgment. We thank Dr. Noriyuki Takada of the Photonics Research Institutes of AIST for help with sample preparations. We also thank Dr. Norio Tanaka of Dainichi Color and Chemicals Manufacturing Co., Ltd., for discussing deeply about the present study.

\section{REFERENCES}

1. C. K. Chiang, Y. W. Park, A. J. Heeger, H. Shirakawa, E. J. Louis, and A. G. McDiarmid, Phys. Rev. Lett., 39, 1098 (1977).

2. P. N. Prasad and D. J. Williams, in "Introduction to nonlinear optical effects in molecules and polymers," Wiley-Interscience, New York, N.Y., 1991, p 235.

3. M. Gailberger and H. Bässler, Phys. Rev. B: Condens. Matter Mater. Phys., 44, 8643 (1991).

4. P. Strohriegl and D. Haarer, Makromol. Chem., Makromol. 
Symp., 44, 85 (1991).

5. M. Berggren, O. Inganas, G. Gustafsson, J. Rasmusson, M. R. Andersson, T. Hjertberg, and O. Wennerstorm, Nature, 372, 44 (1994).

6. A. R. Brown, A. Pomp, C. M. Hart, and D. M. De Leeuw, Science, 270, 972 (1995).

7. J. H. Burroughers, C. A. Jones, and R. H. Friend, Nature, 335, 137 (1998).

8. J. H. Burroughers, D. D. C. Bradley, A. R. Brown, R. N. Marks, K. D. Mackay, R. H. Friends, P. L. Burn, and A. B. Holmes, Nature, 347, 539 (1990).

9. D. Braun and A. J. Heeger, Appl. Phys. Lett., 58, 1982 (1991).

10. R. N. Marks, J. J. M. Halls, D. D. C. Bradley, R. H. Friend, and A. B. Holmes, J. Phys.: Condens. Matter, 6, 1379 (1994).

11. I. Murase, T. Ohnishi, T. Noguchi, M. Hirooka, and S. Murakami, Mol. Cryst. Liq. Cryst., 118, 333 (1985).

12. L. Bakueva, D. Matheson, S. Musikhin, and E. H. Sargent, Synth. Met., 126, 207 (2002).

13. L. Bakueva, E. H. Sargent, R. Resendes, A. Bartole, and I. Manners, J. Mater. Sci.: Mater. Electron, 12, 21 (2001).

14. I. Murase, T. Ohnishi, T. Noguchi, M. Hirooka, and S. Murakami, Polym. Commun., 25, 327 (1984).

15. J. Y. Park, S. B. Lee, T. S. Park, Y. W. Park, C. H. Lee, J. I. Lee, and H. K. Shim, Appl. Phys. Lett., 72, 2871 (1998).

16. H. Mochizuki, T. Mizokuro, N. Yamamoto, N. Tanigaki, T. Hiraga, and N. Tanaka, Jpn. J. Appl. Phys., 42, L613 (2003).

17. T. Mizokuro, H. Mochizuki, M. Xiaoliang, S. Horiuchi, N. Tanaka, T. Hiraga, and N. Tanigaki, Jpn. J. Appl. Phys.,
42, L983 (2003).

18. T. Mizokuro, H. Mochizuki, N. Yamamoto, N. Tanaka, S. Horiuchi, and T. Hiraga, Proc. SPIE, 4991, 333 (2003).

19. H. Mochizuki, T. Mizokuro, N. Tanigaki, and T. Hiraga, Proc. SPIE, 5351, 115 (2004).

20. T. Mizokuro, H. Mochizuki, A. Kobayashi, N. Yamamoto, S. Horiuchi, N. Tanigaki, and T. Hiraga, Chem. Mater., 16, 3469 (2004).

21. H. Mochizuki, T. Mizokuro, N. Tanigaki, X. Mo, and T. Hiraga, Appl. Phys. Lett., 85, 5155 (2004).

22. D. R. Gagnon, J. D. Capistran, F. E. Karasz, and R. W. Lenz, Polym. Bull., 12, 293 (1984).

23. D. D. C. Bradley, J. Phys. D: Appl. Phys., 20, 1389 (1987).

24. C. A. Hunter and J. K. M. Sanders, J. Am. Chem. Soc., 112, 5525 (1990).

25. W. L. Jorgensen and D. L. Severance, J. Am. Chem. Soc., 112, 4767 (1990).

26. S. Tsuzuki, T. Uchimaru, K. Matsumura, M. Mikami, and K. Tanabe, Chem. Phys. Lett., 319, 547 (2000).

27. S. Holdcroft, Adv. Mater., 23, 1753 (2001).

28. T. R. Herbner, C. C. Wu, D. Marcy, M. H. Lu, and J. C. Sturm, Appl. Phys. Lett., 72, 519 (1998).

29. J. Bharathan and Y. Yang, Appl. Phys. Lett., 72, 2660 (1998).

30. D. Braun and A. J. Heeger, Appl. Phys. Lett., 58, 1983 (1991).

31. D. Moses, Appl. Phys. Lett., 60, 3215 (1992).

32. J. H. Ahn, C. Wang, C. Pearson, M. R. Bryce, and M. C. Petty, Appl. Phys. Lett., 85, 1283 (2004). 